\title{
Profiling of Hodgkin's Lymphoma Cell Line L1236 and Germinal Center B Cells: Identification of Hodgkin's Lymphoma-specific Genes
}

\author{
INes SchWering, ${ }^{1,2}$ Andreas BräUninger, ${ }^{3}$ Verena Distler, $^{3}$ JUlia JeSdinsky, $^{2}$ VolKer Diehl, $^{2}$ \\ Martin-Leo HansmanN, ${ }^{3}$ KLAus Rajewsky, ${ }^{1,4}$ and RAlF KÜPPERs ${ }^{1,2}$
}

\begin{abstract}
The malignant cells of classical Hodgkin's lymphoma (cHL), Hodgkin and Reed-Sternberg (HRS) cells, appear to be derived from germinal center (GC) B cells in most cases of the disease. Apart from recent findings of constitutive activation of some transcription factors and autocrine stimulation by cytokine receptors, the mechanisms of malignant transformation in $\mathrm{cHL}$ still remain poorly understood. We performed a large scale gene expression study using serial analysis of gene expression (SAGE), comparing the CHL cell line L 1236 and human GC B cells. Semiquantitative RT-PCR was used to confirm results from the SAGE and to analyze gene expression in 3 additional $\mathrm{CHL}$ cell lines. To investigate expression of some genes in $\mathrm{CHL}$ cases, we applied RT-PCR on microdissected HRS cells. In total, 464 genes showed a change in expression level of 5-fold or higher. For 12 genes (out of 177) identified as upregulated in L1236 cells, RT-PCR confirmed the SAGE results and also showed elevated expression in 3 other $\mathrm{CHL}$ cell lines. For 3 of the upregulated genes, expression by HRS cells in the tissue also was confirmed. Several of the differentially expressed genes may play a role in the pathogenesis of CHL because they represent potential oncogenes, such as rhoC, L-myc, and PTP4A, or transcription factors, such as ATF-5, ATBF1, and p21 SNFT. The genes that showed significantly deregulated expression in HRS cells should be helpful not only for the identification of genes involved in the pathogenesis of $\mathrm{cHL}$ but also for discovering potential prognostic markers or therapeutic targets.
\end{abstract}

\section{INTRODUCTION}

Classical Hodgkin's lymphoma (cHL), one of the most frequent lymphomas in the Western world, is characterized by the presence of rare Hodgkin and Reed-Sternberg (HRS) cells. HRS cells usually represent less than $1 \%$ of the cells in the tumor tissue. They show a peculiar phenotype that does not resemble any normal hematopoietic cell type because coexpression of markers known for distinct hematopoietic lineages is often observed (1-4).

Although HRS cells have lost expression of most typical B cell markers $(1,5,7)$, molecular studies have shown that they derive from B cells in nearly all cases because they carry clonal immunoglobulin gene rearrangements (4). Several findings argue for a derivation of HRS cells from germinal center (GC) B cells: 1. In nearly all cases, HRS cells carry $\mathrm{V}$ region genes modified by somatic hypermutation, a process that takes place specifically in GC B cells $(8,9)$. 2. In several composite Hodgkin and nonHodgkin lymphomas, HRS cells of a cHL are related clonally to the tumor cells of a follicular lymphoma, the paradigm for a GC B cell malignancy (10-12). The presence of shared and distinct somatic $\mathrm{V}$ region gene mutations of HRS and follicular lymphoma cells strongly indicates that these lymphomas derive from 2 distinct daughter cells of a common precursor, a GC B cell.
3. All B cell-derived cHL cell lines have undergone class switch recombination, another process taking place in GC B cells (13). 4. In at least $25 \%$ of cHL cases, HRS cells carry destructive $\mathrm{V}$ gene mutations, leading to the loss of a functional B cell receptor (8). Since GC B cells acquiring such mutations are normally eliminated within the GC by apoptosis, HRS cells likely derive from transformed GC B cells that were rescued from programmed cell death and not from post-GC (that is, memory) B cells (4). In very rare cases (less than 5\%), HRS cells originate from T cells $(14,15)$.

To date, there is no systematic large scale gene expression study available comparing HRS cells with normal GC B cells. In a recent study, a large number of EST clones derived from primary HRS cells and HL cell lines was sequenced (16). However, this analysis was hampered by inclusion of HRS cells of the lymphocyte-predominant subtype of HL, which show many phenotypic differences to HRS cells of cHL (4).

For this reason, we decided to perform serial analysis of gene expression (SAGE) with HRS cells and human GC B cells. The SAGE method is used frequently for comparing gene expression in malignant cells to their nonmalignant counterparts $(17,18)$ because, unlike array-based techniques, it can generate genome-wide expression profiles, including so far unknown transcripts. Because SAGE is technically not yet feasible with primary HRS cells, we

IInstitute for Genetics, 2Department of Internal Medicine I, University of Cologne, Cologne, Germany;

${ }^{3}$ Department of Pathology, University of Frankfurt, Frankfurt/Main, Germany; and ${ }^{4}$ Harvard Medical School, Boston, MA (present address). 
investigated the cHL cell line L1236 with a proven origin from the original HRS cells of a cHL patient $(19,20)$. Although there may be differences in gene expression between cHL cell lines and primary HRS cells, cHL cell lines mirror the gene expression of primary HRS cells in many aspects, not only regarding the expression of specific markers such as TARC, IL-13, and CCR7 $(3,21,22)$ but also in terms of functional aspects such as constitutive NFkB activation (23). By comparing gene expression profiles of cHL cells and their nonmalignant precursor cells, we aimed to identify mechanisms of the pathogenesis of cHL and to identify genes that could represent prognostic markers or therapeutic targets.

\section{MATERIALS AND METHODS}

\section{Cell Lines and Culture}

The cHL cell lines came from patients with cHL of either the nodular sclerosis (L428 and HDLM2) or mixed cellularity (KMH2 and L1236) subtype. HDLM2 comes from T cells; the other 3 HL cell lines come from B cells $(19,20,24)$. All cell lines were grown in RPMI-1640 medium with Glutamax-1 (Gibco, Karlsruhe, Germany) supplemented with $10 \%$ fetal calf serum and $100 \mathrm{U} / \mathrm{mL}$ penicillin/streptomycin at $37^{\circ} \mathrm{C}$ in an atmosphere containing $5 \% \mathrm{CO}_{2}$.

\section{Purification of Human Tonsillar GC B Cells}

Tonsils were obtained from patients after routine tonsillectomy and directly cooled at $4{ }^{\circ} \mathrm{C}$. Mononucleated cells were purified by Ficoll density centrifugation (Amersham, Uppsala, Sweden). CD77 ${ }^{+}$GC B cells were stained consecutively with rat anti-CD77 (Coulter/ Immunotech, Hamburg, Germany), mouse anti-rat IgM (Serotec/ Biozol, Eching, Germany), and anti-mouse IgG1-microbeads (Miltenyi Biotec, Bergisch-Gladbach, Germany) and enriched by magnetic cell separation (Miltenyi Biotec). The purification was performed at $4{ }^{\circ} \mathrm{C}$ to minimize changes in gene expression. In total, $2.5 \times 10^{8} \mathrm{GC}$ B cells were pooled from 8 donors to minimize effects caused by differences in individual gene expression (Figure 1).

\section{Generation and Analysis of SAGE Expression Data}

SAGE generates a quantitative gene expression profile by high throughput sequencing (25). The method is based on the principle that a nucleotide sequence of 10 bases length (a gene tag) usually can be assigned to a unique transcript if its position in the mRNA molecule is known (downstream of the NlaIII restriction site nearest to the poly-A sequence). Poly- $\mathrm{A}^{+} \mathrm{RNA}$ was selected using the Oligotex mRNA kit (Qiagen, Hilden, Germany) and $5 \mu \mathrm{g}$ was used to synthesize cDNA (Gibco). SAGE was performed following the protocol version 1.0d (6) with slight modifications (7). SAGE profiles were generated by sequencing about 30000 tags of both cell populations. The data were analyzed using the SAGE Software 2000, Version 4.12. Adapter tags and duplicate dimers were excluded, and the tag numbers were normalized to 30000 . The differential expression was quantified by generating the quotient of tag numbers of both profiles (for 0 set 1 ), which is given as the factor of down- or upregulation. All genes showing a decrease or increase of 5.0 or higher in expression were included in the analysis. The tag numbers and the quotient of fold change mentioned in text, tables, and figures are rounded up or down.

\section{RNA Isolation and cDNA Synthesis for RT-PCR}

Except for the cDNA samples for GC B cells and cell line L1236, which were synthesized as described above, total RNA of 3 other HL cell lines was isolated with Trizol (Gibco) and treated with DNase I (Promega, Mannheim, Germany). cDNA synthesis was performed using the SuperScript 1st-strand Synthesis System for RT-PCR (Invitrogen, Karlsruhe, Germany).

\section{Semiquantitative RT-PCR}

RT-PCR was performed in duplicates (only 1 of each is shown) using aliquots containing the same amounts of cDNA of the cell line L1236 and GC B cells. cDNA samples were normalized to $\beta$-actin expression by real-time PCR because this procedure allows normalization with high accuracy. $\beta$-actin was chosen because both SAGE profiles show nearly same expression level of $\beta$-actin (Figure 2). For the comparison of the L1236 cell line with GC B cells, the cDNA samples were either pure or 3-, 9-, or 27-fold diluted. RT-PCR was performed in 20 to $50 \mu \mathrm{L}$ of $200 \mu \mathrm{M}$ each of dNTP, $1 \times$ Sigma PCR buffer, $3 \mathrm{mM} \mathrm{MgCl} 2,2$ U Sigma Taq DNA polymerase (Sigma, Deisenhofen, Germany), and $187 \mathrm{nM}$ of each primer. The amplification program consisted of $60 \mathrm{~s}$ at $95^{\circ} \mathrm{C}$, followed by 30 cycles of $20 \mathrm{~s}$ at $95^{\circ} \mathrm{C}, 20 \mathrm{~s}$ at $60^{\circ} \mathrm{C}, 20 \mathrm{~s}$ at $72{ }^{\circ} \mathrm{C}$, and finally $5 \mathrm{~min}$ at $72^{\circ} \mathrm{C}$. The oligonucleotide sequences were as follows: ATBF 1 forward: $5^{\prime}$-GTC TGA CAC GGA TCT CAG C-3', ATBF1 reverse: 5'-GTC CGT TCC TAC ACT GGT C-3', FK506 binding protein 4 forward: 5'-CTG GTT GGA TGG TGG CTT TAG-3', FK506 binding protein 4 reverse: 5'-TTC ATG TGC AAT GGA GGA GG-3', Hnmp1 forward: 5'-TGC TGT CTC TCC TTC ATC CTG T-3', Hnmp-1 reverse: 5'-ATC AAG GCG CCA GTA AAC ACC-3', interleukin-1 receptor associated kinase-1 (IRAK-1) forward: 5'-GGG AAG GGA AAC ATT TTC AGG-3', IRAK-1 reverse: 5'-GGC TAC TTT TGG ACA CGC AAG-3', Jun-B forward: 5'-GGC TCG GTT TCA GGA GTT TGT-3', Jun-B reverse: $5^{\prime}$-GCC CGG ATG TGC ACT AAA AT3', L-myc forward: 5'-TCC ACA CCC GTG AGA AAT CCT-3', L-myc reverse: 5'-TTC CAT ACC CCA TTC CCC A-3', P21 ${ }^{\text {SNFT }}$ forward: 5'-AAG GCT GAC AAG CTC CAT GAG-3', P21 ${ }^{\text {SNFT }}$ reverse: 5'-CCG CAG CAT GGT GTT TTC TT-3', Prame forward: 5'-TGT TTC ATG CCT AAC TAG CTG-3', Prame reverse: 5'-AAC TGT GGC TGC TTT GTT G-3', PTP4A forward: 5'-GAA ATA CCG GCC CAA ACA GAG-3', PTP4A reverse: 5'-TCC TGA CCT ACA TGA CGA CCA G-3', px19 homologue forward: 5'-GAA TTT GGT CTT GCC CGG T-3', px19 homologue reverse: 5'-CGT CTC CTT TGC CTT CTC CTT-3', rhoC forward: 5'-CAG TGC CTT TGG CTA CCT TGA-3', rhoC reverse: 5'-TTG TTC TTG CGG ACC TGG A-3'.

\section{Characteristics of Cases Used for RT-PCR Analysis of Microdissected HRS Cells}

The lymph nodes of $4 \mathrm{cHL}$ patients were analyzed. Patients 1 to 4 were a 29-y-old male with nodular sclerosis HL at relapse, a 40-yold female with 1st presentation of nodular sclerosis HL, a 31-y-old female with 1st presentation of nodular sclerosis HL, and a 33-y-old female with 1st presentation of nodular sclerosis HL, respectively.

\section{Staining of Frozen Sections and Microdissection}

Frozen tissue sections, 5- $\mu \mathrm{m}$ thick, were mounted on membrane-covered slides and incubated with hematoxylin contain- 
A

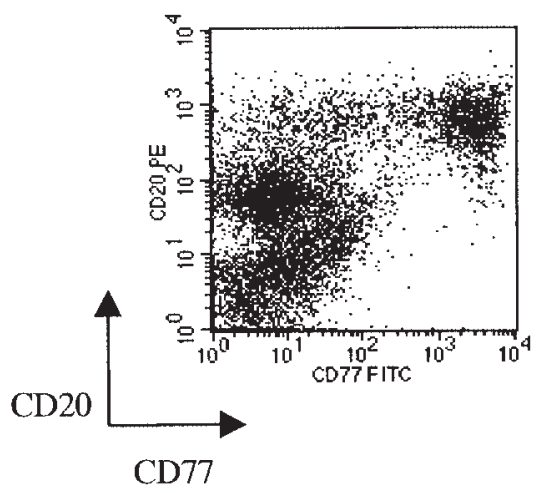

B

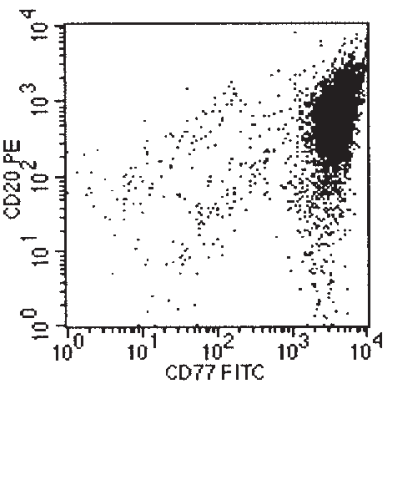

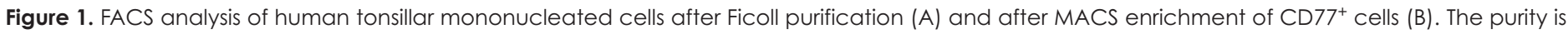
higher than $95 \%$. The figure shows the result for 1 of 8 tonsils used for the SAGE profile.

ing RNase inhibitor (200 U/mL; Roche, Mannheim, Germany) for $4 \mathrm{~min}$. Sections were washed in DEPC-treated water for 2 min, incubated in $2 \%$ eosin for $15 \mathrm{~s}$, washed again, and dried at $37^{\circ} \mathrm{C}$ for $3 \mathrm{~h}$. Single HRS cells were isolated with a UV-Laser beam (P.A.L.M. Microlaser Technologies, Bernried, Germany), catapulted into $20 \mu \mathrm{L}$ of Purescript ${ }^{\circledR}$ lysis buffer (Biozym Group, Hessisch Oldendorf, Germany), and pooled into groups of 50 cells. From the same sections, groups of 5 non-HRS cells were isolated also and pooled into groups of 50 cells. GC B cells were isolated from sections of 2 tonsils and pooled into groups of 50 cells.

\section{RNA Isolation and cDNA Synthesis Using Microdissected Cells}

The Purescript ${ }^{\circledR}$ RNA Isolation Kit (Biozym Group) was applied in a modified manner using glycogen as a carrier and reducing all reagents to one-tenth of the amounts given in the standard protocol. In the analysis of ATBF1 expression a DNase I digestion (Stratagene, Amsterdam, The Netherlands) was performed because of the existence of processed pseudogenes for ATBF1 in the genome. Isolated RNA and $0.6 \mu \mathrm{M}$ of a gene-specific reverse primer (rhoC reverse ${ }^{\mathrm{sc}} 5$ '-CGC TTG TTC TTG CGG ACC TGG A-3', ATBF1 reverse ${ }^{\text {sc }}$ 5'-GGT GTC CGT TCC TCA ACT GGT C$3^{\prime}$, p $21^{\text {SNFT }}$ reverse $2^{\mathrm{sc}} 5^{\prime}$-TCC CGA TCT CTC TCC GCA GCA TGG-3') were heat denatured at $70{ }^{\circ} \mathrm{C}$ for $10 \mathrm{~min}$. T4 gene 32 protein (Ambion, Huntingdon, UK) was added at a concentration of $1.5 \mu \mathrm{g}$ per sample during the initial denaturation. Reverse transcription was performed using the OneStep RTPCR kit components (Qiagen), including 10 U RNase inhibitor (Roche). The reverse transcription reaction mixture was preheated to $50{ }^{\circ} \mathrm{C}$ for $2 \mathrm{~min}$ after which $2 \mu \mathrm{L}$ of Qiagen OneStep RT-PCR enzyme mix was added. cDNA synthesis was performed at $50{ }^{\circ} \mathrm{C}$ for $30 \mathrm{~min}$.

For several samples of tonsillar GC B cells, the presence of intact RNA was verified by RT-PCR analysis for CD52 transcripts. CD52 is expressed by all lymphocytes. For reverse transcription, the primer CD52 reverse (5'-CTG GTG ATG TCT GGC ATC AAC C-3') was used under the conditions described above.

\section{PCR Amplification of cDNA Products from Microdissected Cells}

For RT-PCR analysis, 2 rounds of PCR were performed, using $1 \mu \mathrm{L}$ of the 1 st round reaction in the 2 nd round. All primer pairs except for ATBF1 were intron-spanning. In all reactions, dNTP concentrations were $200 \mu \mathrm{M}$, primer concentrations were $0.125 \mu \mathrm{M}$, and the HotStarTaq DNA polymerase system (Qiagen) was used in a reaction volume of $50 \mu \mathrm{L}$. The 1 st round of PCR was performed applying the reverse primers used for cDNA synthesis and the corresponding forward primers (rhoC forward $^{\text {sc }} 5^{\prime}$-ACC CGG ACA CTG ATG TCA TCC TC-3', ATBF1 forward $^{\text {sc }} 5^{\prime}$-CCT CCA GGA AGT CTT GGC CGC-3', p21 ${ }^{\text {SNFT }}$ forward $^{\text {sc }}$ 5'-AGC TCC CGG CAT GTC GCA AGG-3'). For amplification of a fragment of the rhoC and the ATBF1 genes, samples were adjusted to $3 \mathrm{mM} \mathrm{MgCl} 2$. Cycling conditions were $15 \mathrm{~min}$ at $95^{\circ} \mathrm{C}, 60 \mathrm{~s}$ at $61^{\circ} \mathrm{C}(\mathrm{ATBF} 1)$ or $65^{\circ} \mathrm{C}$ (rhoC), $60 \mathrm{~s}$ at $72{ }^{\circ} \mathrm{C}, 24$ cycles (ATBF1) or 29 cycles (rhoC) of $30 \mathrm{~s}$ at $95^{\circ} \mathrm{C}, 30 \mathrm{~s}$ at $61{ }^{\circ} \mathrm{C}$ (ATBF1) or $65^{\circ} \mathrm{C}$ (rhoC), and $45 \mathrm{~s}$ at $72{ }^{\circ} \mathrm{C}$ followed by a final $5 \mathrm{~min}$ incubation at $72{ }^{\circ} \mathrm{C}$. In the 2 nd round of amplification, an internal forward primer (rhoC forward2 $2^{\text {sc }} 5^{\prime}-\mathrm{CAG}$ TGC CTT TGG CTA CCT TGA-3'; ATBF1 forward2sc 5'-GGA GTC TGA CAC GGA TCT CAG C-3') was used together with the reverse primer of the 1st round to amplify a $115 \mathrm{bp}$ (ATBF1) or a $105 \mathrm{bp}$ (rhoC) fragment of the genes. The reaction and cycling conditions were the same as in the 1st round, with the exceptions that either 27 or 30 cycles were performed (rhoC), $2 \mathrm{mM}$ $\mathrm{MgCl}_{2}$ was used, and the annealing temperature was $65{ }^{\circ} \mathrm{C}$ (ATBF1). For amplification of a fragment of the p21 ${ }^{\mathrm{SNFT}}$ gene, samples were adjusted to $2 \mathrm{mM} \mathrm{MgCl}{ }_{2}$. Cycling conditions were $15 \mathrm{~min}$ at $95^{\circ} \mathrm{C}, 60 \mathrm{~s}$ at $65^{\circ} \mathrm{C}, 60 \mathrm{~s}$ at $72{ }^{\circ} \mathrm{C}$, and 29 cycles of $30 \mathrm{~s}$ at $95^{\circ} \mathrm{C}, 30 \mathrm{~s}$ at $65^{\circ} \mathrm{C}$, and $45 \mathrm{~s}$ at $72{ }^{\circ} \mathrm{C}$, followed by a final $5 \mathrm{~min}$ incubation at $72{ }^{\circ} \mathrm{C}$. In the 2 nd round of amplification, an internal reverse primer $\left(\mathrm{p} 21^{\mathrm{SNFT}}\right.$ reverse $1^{\mathrm{sc}} 5^{\prime}$-TTC GGA CCT TCC TGT CAT CAT CC-3') was used together with the forward primer of the 1st round to amplify a 131-bp fragment of the gene. The reaction and cycling conditions were the same as in the 1 st round, with the exception that $1.5 \mathrm{mM} \mathrm{MgCl}_{2}$ was used. Some PCR products were sequenced, confirming that the correct products were amplified. 


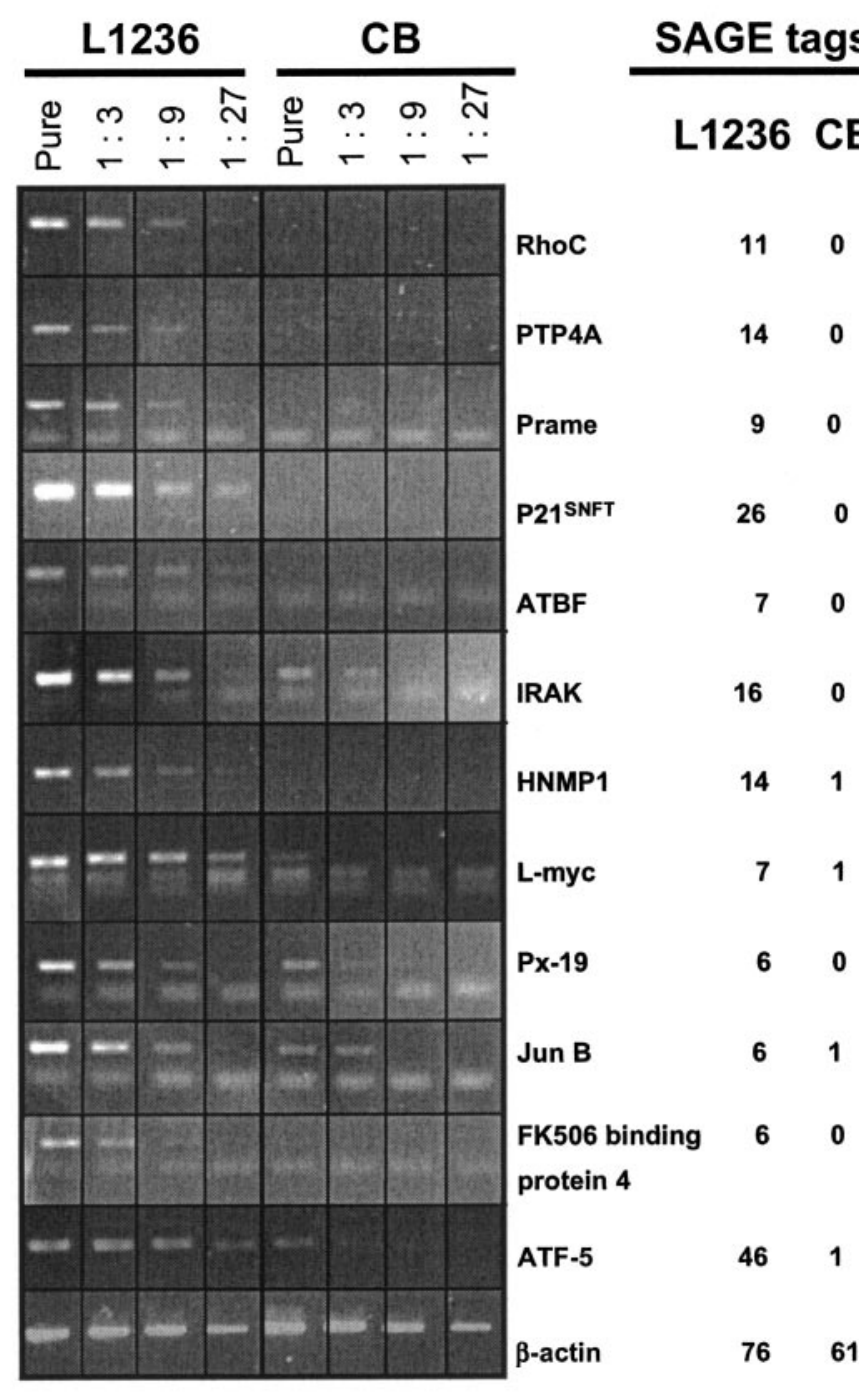

Figure 2. Semiquantitative RT-PCR analysis for 12 genes in L1236 and GC B cells (CB, centroblasts). CDNA of L1236 and GC B cells was normalized to $\beta$-actin by real-time PCR. Equal amounts of CDNA were introduced in RT-PCR analysis using 4 different CDNA dilutions for L1236 (left panel) and GC B cells (right panel). RT-PCR was performed until a faint band was detectable in the lowest dilution of the L1236 CDNA. By comparing the intensity of the bands of $L 1236$ and GC B cells on an agarose gel, the difference of expression for a given gene was estimated. Normal RT-PCR analysis of $\beta$-actin is given in the lowest panel and shows the same amount of PCR product for both cell populations. In total, 12 genes found to be upregulated in the SAGE analysis were analyzed. For some cDNAs primerdimers are visible and represent the lower bands. Tag numbers are normalized to 30000 tags.

Samples of HRS cells and control cells were analyzed in parallel, together with controls containing only Purescript ${ }^{\circledR}$ lysis buffer. Some non-HRS cell samples were analyzed in parallel with $\mathrm{KMH} 2$ positive controls.

PCR for transcripts of the CD52 gene from GC B cell samples was performed in 1 round of PCR (40 cycles), using the cDNA synthesis primer and a forward primer binding to a sequence in exon 1 of the gene (CD52Ex1, 5'-GCT GCT ACC AAG ACA GCC ACG-3'). PCR conditions were as described above, using $2 \mathrm{mM}$
$\mathrm{MgCl}_{2}$ and $65{ }^{\circ} \mathrm{C}$ annealing temperature. The resulting intronspanning PCR product has a length of $327 \mathrm{bp}$. A PCR product of expected length was obtained from 5 of 6 samples tested.

\section{RESULTS}

Generation and Comparison of SAGE Profiles from the cHL Cell Line L1236 and GC B Cells

We generated SAGE profiles from the cHL cell line L1236 and human GC B cells. For the isolation of GC B cells we decided to use $\mathrm{CD}^{+} 7^{+} \mathrm{GC}$ cells because gene expression analysis based on oligonucleotide arrays (26) showed surprisingly few differences between gene expression patterns of tonsillar centrocytes (defined as $\mathrm{CD}^{+} \mathrm{CD}^{+}{ }^{-}$) and tonsillar centroblasts (defined as $\mathrm{CD}^{+} 7^{+}$) (27). The reason for this may be that CD77 does not completely distinguish between these 2 cell populations, although CD77 is commonly used as a centroblast marker. The purity of $\mathrm{CD}^{+} 7^{+}$cells used for this study was higher than 95\% (see Figure 1).

After exclusion of duplicate dimers and adapter tags, a total of 29514 tags for the GC B cell profile and 30034 tags for the CHL cell line profile were analyzed and represented 11056 and 10493 distinct tags, respectively. The abundance class distribution is as expected from previous SAGE studies. Most transcripts (83\%) are represented with 1 or 2 tags, whereas $8 \%$ of transcripts are present with 5 tags or more.

In total, 464 distinct transcripts were shown to be differentially expressed by a factor of 5 or higher. The factor of 5 was chosen as an arbitrary cut-off point in this analysis, due to the high number of differentially expressed genes identified. It should be pointed out, however, that this led to the exclusion of a significant number of genes that may be important in cHL pathogenesis, as they are expressed in L1236 at high levels and are expressed more strongly in L1236 than in GC B cells. (A list of these genes is available from the authors upon request.) Moreover, if larger SAGE profiles had been generated, additional differentially expressed genes that were below our cut-off point of 5-fold difference would have been identified.

When compared with GC B cells, the cell line L1236 showed 177 transcripts with higher expression and 287 with lower expression. (The complete list of differentially expressed tags [5-fold or higher] is available online at http://www.bloodjournal.org/cgi/ content/full/101/4/1505/DC1.) The 20 transcripts with the highest decrease or increase in expression are shown in Tables 1 and 2. Several genes previously reported to be specifically expressed or upregulated in HRS cells were found to be upregulated in this study also, namely the actin-bundling protein Fascin (18 tags in L1236:0 tags in GC B cells), the chemokine TARC (14:0), TNF (8:1), CD44 (6:0), neuron-specific enolase 2 (8:1), and CCR7 (18:0) whereas a few other genes, such as IL-13 and CD30, were not identified $(2,3,19,21,22,28-30)$. The latter could be due to a relatively low level of gene expression or the presence of gene polymorphisms in the tag sequences of L1236, which is known to be positive for both CD30 and IL-13 $(19,22)$.

\section{Identification of Downregulated Transcripts in L1236}

Analyzing the downregulated genes, a dramatic loss of B lineage-specific gene expression became obvious, affecting 
Table 1. The 20 transcripts displaying the largest decrease in expression in the cell line L1236 compared with GC B cells

\begin{tabular}{|c|c|c|c|c|}
\hline \multirow[b]{2}{*}{ Fold difference } & \multirow[b]{2}{*}{ Tag sequence } & \multicolumn{2}{|c|}{$n$} & \multirow[b]{2}{*}{ Unigene cluster: gene name } \\
\hline & & $\mathrm{CB}$ & L1236 & \\
\hline 79 & AAGGGAGCAC & 79 & 0 & Hs.181125: Ig lambda \\
\hline 58 & GGGCATCTCT & 58 & 1 & Hs.76807: MHC class II, DR alpha \\
\hline 39 & ATGAGTGCTG & 39 & 0 & Hs.86693: EST \\
\hline 39 & TTGGTGAAGG & 154 & 4 & $\begin{array}{l}\text { Hs.288031: sterol-C5-desaturase-like } \\
\text { Hs.75968: thymosin, } \beta 4\end{array}$ \\
\hline 37 & TGTACCCCGC & 37 & 0 & Hs.155975: protein tyrosine phosphatase \\
\hline 36 & GACCCAACTG & 36 & 1 & Hs.89575: CD79B \\
\hline 34 & GCGGTTGTGG & 34 & 1 & Hs.79356: LAPTm5 \\
\hline 33 & AAACCCCAAT & 33 & 0 & $\begin{array}{l}\text { Hs.348136: Homo sapiens mRNA } \\
\text { Hs.77735: hypothetical protein FLJ } 11618\end{array}$ \\
\hline 32 & GGGGCAACAG & 32 & 0 & $\begin{array}{l}\text { Hs.276770: CDW52 (CAMPATH-1) } \\
\text { Hs.29288: hypothetical protein FLJ21865 }\end{array}$ \\
\hline 27 & AAACCAGAGG & 27 & 1 & $\begin{array}{l}\text { Hs.40202: Iymphoid-restricted membrane protein } \\
\text { Hs.4291: golgi phosphoprotein } 5\end{array}$ \\
\hline 26 & GCAGTGGGAA & 26 & 0 & Hs.890: Iymphotoxin $\beta$ \\
\hline 24 & TCTCTCAAAG & 24 & 0 & Hs.82212: CD53 \\
\hline 23 & ACTTTTTCAA & 140 & 6 & $\begin{array}{l}\text { Hs.136824: ESTs } \\
\text { Hs.156814: KIAA0377 gene product }\end{array}$ \\
\hline 22 & TTTCAATAGA & 22 & 0 & $\begin{array}{l}\text { Hs.288025: Homo sapiens CDNA } \\
\text { Hs.50601: hypothetical protein MGC10986 }\end{array}$ \\
\hline 21 & GCAGTTCTGA & 21 & 0 & $\begin{array}{l}\text { Hs.308026: MHC class II, DR } \beta 5 \\
\text { Hs.349123: MHC, class II, DR } \beta 1\end{array}$ \\
\hline 20 & TATGAGGACA & 20 & 0 & Hs.79630: CD79A \\
\hline 19 & CTGACTGTCC & 19 & 1 & Hs.84298: CD74 \\
\hline 18 & GGACCAGGCT & 18 & 0 & Hs.62771: Homo sapiens mRNA \\
\hline 18 & TTAAATCCCA & 18 & 1 & Hs.170121: CD45 \\
\hline 18 & ACGCTCTCGA & 18 & 1 & Hs.153053: CD37 \\
\hline
\end{tabular}

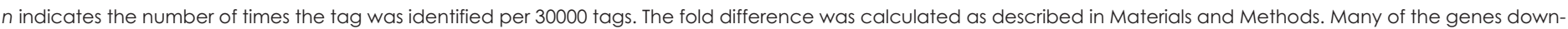

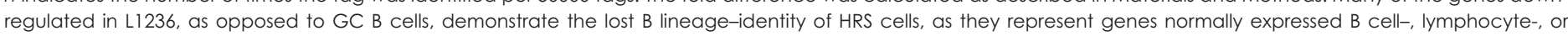
hematopoietic-specifically and are presented in detail elsewhere (7). CB, centroblasts.

nearly all B-cell specific genes. Only some genes involved in MHC class II-restricted antigen presentation are still expressed or even show an elevated expression, for example, CD40, B7.1, B7.2, and some MHC class II alleles. A detailed analysis and discussion regarding this phenotypic loss of B lineage identity is presented elsewhere (7). In addition to the B lineage-specific genes, the most highly decreased transcripts include the regulator of G-protein signaling 13, a negative regulator of G-protein signaling (31) that is 14-fold downregulated in L1236. Notably, 2 other transcripts that function in negative regulation of G-protein signaling also are downregulated in HRS cells: regulator of Gprotein signaling 5 (11-fold) (32) as well as G-protein pathway suppressor 1 (6-fold) (33). Another, 2 transcripts with an inhibiting function for rho protein activity show reduced expression in cell line L1236, namely rho GDP dissociation inhibitor (GDI) $\alpha$ and rho (GDI) $\beta$ (both 6-fold) $(34,35)$.

\section{Identification of Upregulated Genes in L1236}

Our further studies concentrated on transcripts showing an increased expression in the cHL cell line L1236. We excluded from the analysis all tags lacking an entry in Unigene, matching not further specified cDNAs or protein Genbank entries, and genes that function in more general metabolic processes. From the remaining genes with known function and potential functional interest, we selected 46 genes that we considered to be of particular interest and divided them into functional classes, such as transcription factors or kinases and phosphatases (Table 3). Several transcripts known to be involved in or associated with malignant transformation are upregulated or specifically expressed in the L1236 cell line. This includes potential oncogenes, such as PTP4A (36), rhoC (37), or L-myc (38), cathepsins known to be important in tumor progression (39), and genes involved in cell adhesion, such as the laminin receptor (40) or CD44 (41). In addition to molecules playing a role in signaling cascades, such as Dvl-1 (42) or Nm23-H1 (43), several transcription factors show elevated expression (see Table 3).

\section{Semiquantitative RT-PCR Verifies SAGE Results}

To investigate the reliability of SAGE results, selected upregulated genes were analyzed by semiquantitative RT-PCR for their expres- 
Table 2. The 20 transcripts displaying the strongest increase in expression in the cell line L1236 compared with GC B cells

\begin{tabular}{|c|c|c|c|c|}
\hline \multirow[b]{2}{*}{ Fold difference } & \multirow[b]{2}{*}{ Tag sequence } & \multicolumn{2}{|c|}{$n$} & \multirow[b]{2}{*}{ Unigene cluster: gene name } \\
\hline & & CB & L1236 & \\
\hline 74 & GTCCCTGCCT & 1 & 74 & Hs.301961: glutathione S-transferase \\
\hline 46 & AAATGTTTGG & 1 & 46 & Hs.9754: ATF-5 \\
\hline 38 & CCCTGGGTTC & 7 & 266 & Hs. 111334 : ferritin, light polypeptide \\
\hline 36 & TACAGCACGG & 0 & 36 & Hs.111811: microsomal glutathione S-transferase 3 \\
\hline 35 & TCACTCCTGG & 1 & 35 & $\begin{array}{l}\text { Hs.75859: mitochondrial ribosomal protein L } 49 \\
\text { Hs.9754: ATF-5 }\end{array}$ \\
\hline 30 & TGGGGTTCTT & 0 & 30 & $\begin{array}{l}\text { Hs.272499: alcohol dehydrogenase family member } \\
\text { Hs.62954: ferritin, heavy polypeptide } 1\end{array}$ \\
\hline 26 & GAGGCCACCC & 0 & 26 & Hs.62919: p21SNFT \\
\hline 20 & ACTGCAGCCA & 0 & 20 & $\begin{array}{l}\text { Hs.22404: protease, serine, } 12 \\
\text { Hs.67726: MARCO }\end{array}$ \\
\hline 18 & GTGCCATATT & 1 & 18 & $\begin{array}{l}\text { Hs.254709: EST } \\
\text { Hs.5337: isocitrate dehydrogenase } 2\end{array}$ \\
\hline 18 & TTCTGCTTTC & 0 & 18 & Hs.1652: CCR7 \\
\hline 18 & GATACAGCCA & 1 & 18 & Hs.1416: CD23A \\
\hline 18 & GCAAACGTTG & 0 & 18 & Hs.49768: ESTs \\
\hline 18 & TGCAGGCCTG & 1 & 18 & Hs.82030: tryptophanyl-tRNA synthetase \\
\hline 17 & GCCCCCAATA & 1 & 17 & Hs.227751: galectin 1 \\
\hline 17 & ATAGTAGCTT & 0 & 17 & Hs. $118400:$ Fascin \\
\hline 17 & GTGACCACGG & 1 & 17 & $\begin{array}{l}\text { Hs.299882: ESTs, Highly similar to N-methyl-D- } \\
\text { aspartate receptor 2C subunit precursor }\end{array}$ \\
\hline 16 & CTCAACСССС & 0 & 16 & $\begin{array}{l}\text { Hs.89137: low density lipoprotein related protein } 1 \\
\text { Hs.234249: MAPK } 8 \text { interacting protein } 1\end{array}$ \\
\hline 16 & CCCCCGTGAA & 0 & 16 & Hs.182018: IRAK-1 \\
\hline 16 & CCTGGAAGAG & 1 & 16 & Hs.75655: thyroid hormone binding protein p55 \\
\hline 15 & TTTTCTGAAA & 0 & 15 & Hs.76136: thioredoxin \\
\hline
\end{tabular}

$n$ indicates the number of times the tag was identified per 30000 tags. The fold difference was calculated as described in Material and Methods. CB, centroblasts.

sion levels. We chose 12 of the 46 genes that we found to be of potential functional relevance for cHL pathogenesis (see Table 3) for this analysis: Prame, a common tumor antigen (44); PTP4A, a putatively oncogenic phosphatase (36); p21 2 NFT, a Jun-dimerization protein which inhibits AP-1-mediated transactivation (45); rhoC, a member of the Ras homology family, which plays a role in transformation and tumor progression (37); ATF-5, a transcription factor of the ATF/CREB protein family (46); ATBF1, a transcription factor containing zinc finger and homeobox domains (47); L-myc, a transcription factor of the myc protein family (38); IRAK-1, which plays a role in IL-1/IL-18 and Toll-like receptor signaling (48); Hnmp-1, a transmembrane protein with potential receptor function (49); px19 homologue, a transcription factor with unknown function (50); FK506 binding protein 4 , an immunophilin which binds to the imunosuppressant FK506 and is ubiquitously expressed (51), and Jun-B, a transcription factor of the basic leucine zipper protein family (52).

Equal amounts of cDNA were introduced in RT-PCR analysis. Four cDNA dilutions were used to facilitate a semiquantitative estimation of the differences in expression levels between L1236 cells and GC B cells (Figure 2). For most of the investigated transcripts, the difference in expression between the cell line L1236 and GC B cells was verified (see Figure 2). There are some differences regarding the factor of differential expression. Hnmp-1, ATF-5, and px19.
These differences can be easily explained by the fact that all 3 transcripts have low expression (at least in GC B cells) and therefore were represented in the SAGE profiles with low tag numbers. In such cases, levels of transcription predicted by SAGE are obviously not as reliable as they are for more highly expressed genes.

Meanwhile we also have used, in collaboration with R DallaFavera's group, oligonucleotide microarrays to monitor gene expression in HL cell lines (53). While a global comparison of these and the SAGE data is difficult because appropriate software is not available, we have found that of the 12 genes shown by SAGE and RT-PCR to be upregulated in the L1236 cell line, 10 also have shown higher expression in L1236 than in human centroblasts in the microarray analysis. The remaining 2 genes (px19homologue and $\mathrm{P} 21^{\mathrm{SNFT}}$ ) were not present on the chip.

\section{Analysis of Gene Expression in 4 HL-derived Cell Lines: Expression of 12 Selected Genes as a General Feature of HL Cell Lines}

Are the genes upregulated in the L1236 cell line generally upregulated in HRS cells? To investigate this issue, we performed RT-PCR for the 12 genes described above, comparing semiquantitatively the expression levels in L1236 and 3 other cHL cell lines (L428, KMH2, and HDLM2). PCR results show that all transcripts 
Table 3. Transcripts with an increased expression

Functional classes ${ }^{a}$

Gene function

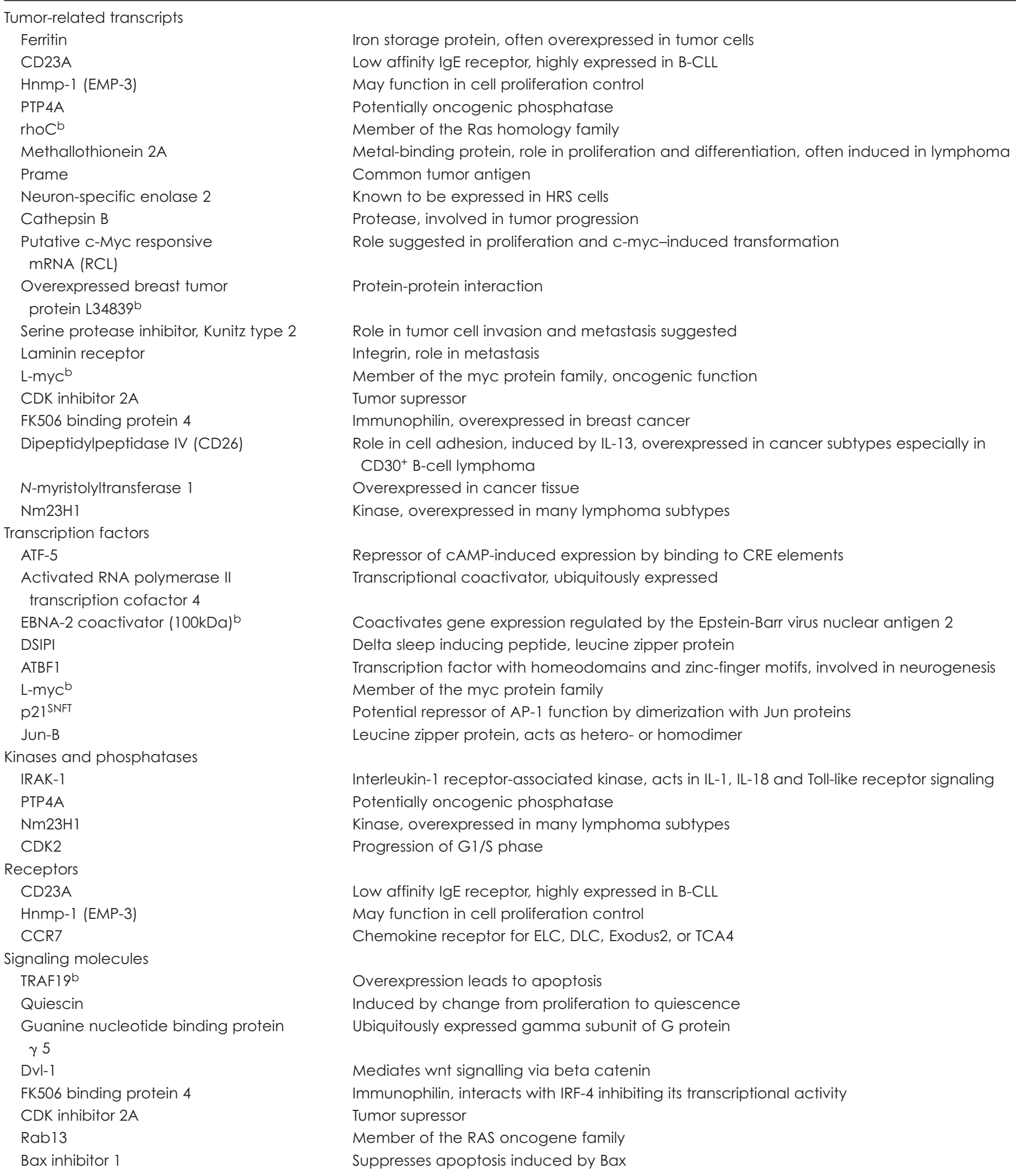


Table 3. Continued

Functional classes

Gene function

\author{
Adhesion \\ Galectin 1 \\ Laminin receptor \\ Dipeptidylpeptidase IV (CD26) \\ CD44 \\ Chemokines, cytokines \\ TARC \\ TNF member 2 \\ Interferon-induced transcripts \\ Interferon-induced 15-kd protein \\ Complement component C4A \\ Proteasome activator subunit 2, hPA28 \\ Interferon-inducible transmembrane \\ protein 1 \\ Interferon-induced transcription \\ factor $3, \gamma$ \\ Transcripts previously known to be highly \\ expressed in HRS cells \\ Fascin \\ TARC \\ Topoisomerase II $\alpha$ \\ TNF member 2 \\ CD44
}

Neuron-specific enolase 2

Cathepsin B
Implication in modulating cell-cell and cell-matrix interaction

Integrin, role in metastasis

Role in cell adhesion, induced by IL-13, overexpressed in cancer subtypes especially in $\mathrm{CD} 30^{+} \mathrm{B}-\mathrm{cell}$ lymphoma

Stimulation of CD44 augments the LFA-1-mediated adhesion of cancer cells to endothelial cells and induces expression of c-Met, known to be expressed in HRS cells

T-cell attractance

Tumor necrosis factor, induces apoptosis or proliferation dependent on the target cell

Function not known

Esterase in the complement cascade, upregulated by interferon gamma

Positive effector of the 20 S proteasome, Inducible by interferon- $\gamma$ and other cytokines

Involved in relaying antiproliferative signals

Activates transcription in response to interferon $\alpha$

Dendritic cell marker

T-cell attractance

Expression associated with proliferation

Tumor necrosis factor, induces apoptosis or proliferation dependent on target cell

Stimulation of CD44 augments the LFA-1-mediated adhesion of cancer cells to endothelial

cells and induces expression of c-Met

Known to be expressed in HRS cells

Protease, involved in tumor progression

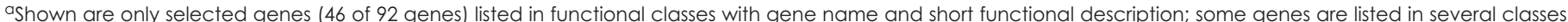

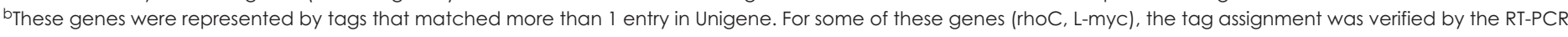
results (see Figure 2).

analyzed can be detected in all $4 \mathrm{HL}$ cell lines, albeit in different amounts (Figure 3). For most transcripts, expression levels in L428, KMH2, and HDLM2 cell lines are as high as or higher than in L1236 (see Figure 3). Therefore, the genes identified in this study likely represent transcripts that are generally upregulated in HL cell lines compared with human GC B cells.

\section{Analysis of Primary cHL Cases by RT-PCR of Microdissected HRS Cells}

Because RT-PCR on primary microdissected HRS cells is laborintensive, only 3 of the 12 genes shown to be expressed in all 4 cHL cell lines (rhoC, ATBF1, and p21 ${ }^{\mathrm{SNFT}}$ ) were exemplarily analyzed for expression in primary HRS cells. Nearly 2000 single microdissected HRS cells from 4 cases of cHL were pooled into groups of 50 cells. Three such samples per cHL case were analyzed by a seminested RT-PCR approach in parallel with groups of 50 non-HRS cells (mainly lymphocytes) from the same sections and groups of 50 centroblasts from 2 tonsils (Table 4). For each of the 3 genes, all 3 or at least 2 samples of HRS cells from the 4 cases analyzed were positive in the PCR, whereas nearly all samples from non-HRS cells and all GC B cells were negative (see Table 4). Thus, we confirmed expression of 3 genes in primary HRS cells.

\section{DISCUSSION}

Reliability of SAGE Analysis and Its Relevance for the Identification of HRS Cell-specific Genes

In the present study, large scale gene expression profiles were generated to identify genes specifically expressed or upregulated in HRS cells compared with human GC B cells, which represent their nonmalignant counterpart in nearly all cases of cHL. L1236, an HRS cell line, was used for this purpose. Its GC B cell origin is suggested by the nature of the Ig gene rearrangements in its genome $(20,54)$. However, a potential limitation of the gene expression comparison lies in the adaptation of the cells to growth in culture, which likely affects their gene expression pattern. With this reservation in mind, it is encouraging that several genes previously reported to be specifically expressed or upregulated in HRS cells were identified as upregulated in the L1236 cell line (see Results). The identification of these markers not only argues for 


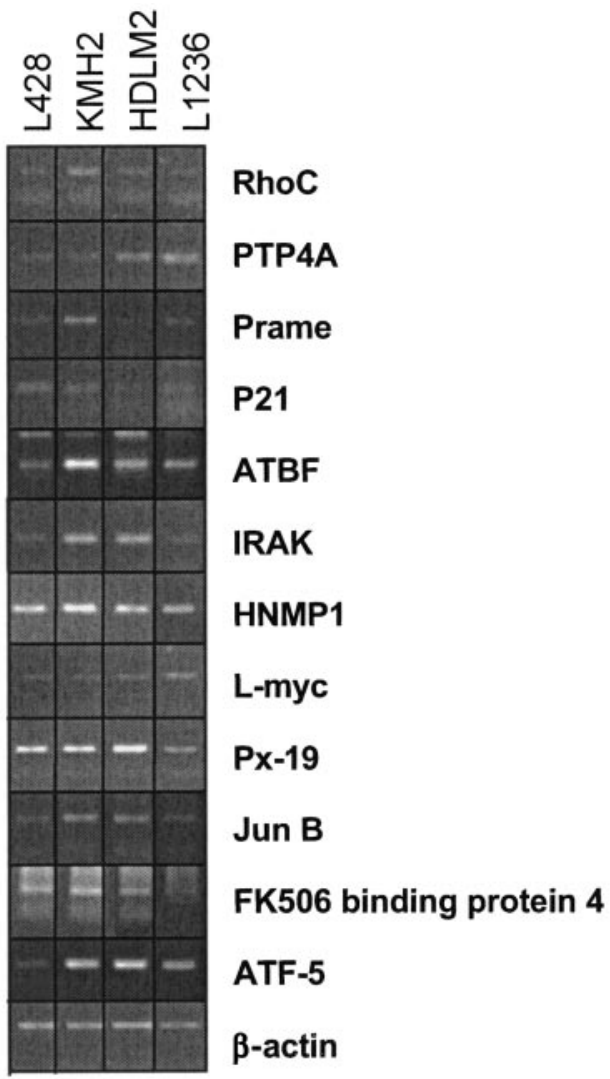

Figure 3. Semiquantitative RT-PCR analysis for 12 genes in $4 \mathrm{HL}$ cell lines (L428, KMH2, HDLM2, and L1236). The CDNA of the $4 \mathrm{HL}$ cell lines was normalized to $\beta$-actin by real-time PCR. RT-PCR analysis of $\beta$-actin is given in the lowest panel and shows the same amount of PCR product for all cell populations. A limited cycle number was performed to retain the differences in band intensities in the gel analysis. For each gene, the PCR results are shown for the lowest cycle number at which a PCR product was obtained for all 4 cell lines. These cycle numbers were between 28 and 35 cycles. For some cDNAs primerdimers are visible and represent the lower bands. For 2 transcripts, PTP4A and L-myc, L1236 is the cell line which shows the highest expression of the $4 \mathrm{HL}$ cell lines. Therefore, for these genes it cannot clearly be stated that the expression level in the 3 other $\mathrm{HL}$ cell lines is much higher than that in GC B cell because GC B cells were not included in this analysis.

the reliability of SAGE to identify differentially expressed genes but also argues for the value of HL cell lines in reflecting the gene expression of primary HRS cells

The main advantage of the SAGE technique is the quantitative nature of the results. To confirm this, we performed semiquantitative RT-PCR for 12 transcripts identified as upregulated in L1236. The PCR results verified the upregulation shown by SAGE for all genes tested. To exclude the possibility that only L1236-specific rather than HL-specific genes were identified, these 12 genes were analyzed for expression in 3 other HL cell lines. By semiquantitative RT-PCR, transcripts of all 12 genes were detected in these $3 \mathrm{HL}$ cell lines. Therefore, upregulation of these genes seems to be a common feature of HL cell lines.

Unfortunately, no commercially available antibodies exist for many of these genes and analysis with anti-rhoC and anti-L-myc antibodies failed to give evaluable results on immunoblot analy- sis. However, for 3 genes (rhoC, ATBF1, and p21 ${ }^{\mathrm{SNFT}}$ ), expression by primary HRS cells was confirmed by RT-PCR on microdissected HRS cells from 4 cases of cHL (see Table 4). This indicates that at least a significant fraction of the genes identified here are also expressed in primary HRS cells.

While the present analysis is based on the comparison of cHL cells to their normal counterpart, it also would be interesting to analyze SAGE profiles of non-Hodgkin lymphomas for the expression of the genes identified here as being differentially expressed between HL and GC B cells. Such profiles have not yet been published, but SAGE allows one to perform comparisons with other profiles once they become available.

\section{Downregulated Transcripts in L1236 Compared with GC Cells}

In addition to the downregulation of most B lineage genes (see Results), the strong downregulation of 3 inhibitors of G-protein signaling is of interest, namely regulator of G-protein signaling 5 (32) and 13 (31) and G-protein pathway suppressor 1 (33), although another inhibitor, regulator of G-protein signaling 16 (55), shows nearly 5-fold upregulation. Decreased expression of these inhibitors could influence G-protein signaling in HRS cells. Two transcripts coding for rho GDI $\alpha$ and $\operatorname{GDI} \beta(34,35)$, which are negative regulators of Rho protein activity, also showed decreased expression in L1236. This finding is of special interest because rho genes represent oncogenes and rhoC is shown here to be overexpressed in HRS cells (see below). Whether the lack of these inhibitory proteins results in an enhanced activity of the corresponding pathways in HRS cells remains to be determined.

Table 4. RT-PCR analysis of microdissected HRS cells for expression of rhoC, ATBF1, and $\mathrm{p} 21^{\text {SNFT }}$

Samples positive/samples analyzed

\begin{tabular}{lccc} 
Samples $^{a}$ & rhoC & ATBF1 & p21 SNFT $^{\text {SN }}$ \\
\hline HRS cells & $3 / 3$ & $3 / 3$ & $3 / 3$ \\
Case 1 & $3 / 3$ & $3 / 3$ & $2 / 3$ \\
Case 2 & $3 / 3$ & $3 / 3$ & $3 / 3$ \\
Case 3 & $3 / 3$ & $3 / 3$ & $2 / 3$ \\
Case 4 & & & \\
Controls (cases 1 to 4) & $6 / 6$ & $5 / 5$ & $5 / 6$ \\
KMH2 cells & $3 / 19$ & $1 / 12$ & $0 / 12$ \\
Non-HRS cells & $0 / 6$ & $0 / 6$ & $0 / 6$ \\
GC B cells & $0 / 14$ & $0 / 12$ & $1 / 12$ \\
Buffer controls & & & \\
\hline
\end{tabular}

aGroups of 50 HRS cells and 50 non-HRS cells (small cells isolated from the same tissue sections as the HRS cells) were analyzed. Pools of $50 \mathrm{GC} \mathrm{B}$ cells isolated from sections of 2 tonsils were analyzed as additional controls. Intact RNA in the tonsillar samples was confirmed by successful amplification of a fragment of the CD52 RNA from several GC B-cell samples (not shown). Buffer controls represent reaction tubes with buffer but without cells. Groups of 10 FACS-sorted KMH2 cells served as positive controls for CDNA synthesis and RT-PCR.

bThe few positive non-HRS cell samples may be due to cellular contamination of the samples by fragments from HRS cells or expression of the respective genes by some cells among the non-HRS cells. 


\section{Upregulated Transcripts in L1236 Compared with GC B Cells}

In total, 178 transcripts showed at least 5-fold upregulation in cell line L1236. Table 3 lists 46 of what we considered to be the most interesting genes. Twelve of these genes, all of which were shown to be expressed in all cHL cell lines analyzed, and 3 which were expressed in primary HRS cells also, are discussed in more detail in the following paragraph.

Several of the upregulated genes represent (putative) oncogenes: rhoC is a member of the Ras homologous (Rho) subfamily of proteins, which functions in actin-remodeling processes. Rho proteins are involved in cell cycle progression, differentiation processes, and transformation (56). In addition, rhoC was shown to play an important role in tumor progression as overexpression enhances metastasis (37). The expression of rhoC in HRS cells in cHL tissues (see Table 4) renders it an interesting candidate for further investigations. Protein phosphatase IVA (PTP4A or Prl-3) itself has not yet been shown to act as an oncogene, but Prl-1 and Prl-2, close homologues of PTP4A, can transform mouse fibroblasts and hamster pancreatic epithelial cells $(57,58)$. Moreover, high expression levels of PTP4A correlate with metastasis in colorectal cancer (36). L-myc is a well-known oncogene of the myc gene family (38).

In addition to L-myc, 4 other transcription factors showed an elevated or specific expression in cHL cell lines: ATF-5, ATBF1, p21 2 SFT, and Jun-B. ATF-5, a member of the ATF/CREB protein family, is supposed to regulate cAMP-induced expression and was shown to bind cAMP-responsive elements as a homodimer (46). ATBF1, originally identified as a repressor of the $\alpha$-fetoprotein gene (47), also acts as a repressor of c-myb transcriptional activity by protein-protein interaction (59). Additionally, ATBF1 plays a role in myogenic and neurogenic differentiation $(60,61)$. Another transcription factor strongly upregulated in L1236 is the Jun dimerization protein $\mathrm{p} 21^{\mathrm{SNFT}}$, which was shown to repress AP-1 activity, probably by forming heterodimers with c-Jun (45).The role of p21 ${ }^{\mathrm{SNFT}}$ in HRS cells is not clear however, because at least some AP-1 target genes are expressed and inhibition of AP-1 leads to decreased proliferation in these cells (62). Jun-B, slightly upregulated in L1236 compared with GC B cells, is also a member of the basic leucine zipper protein family but in contrast to c-jun no significant transforming capacity has been shown so far (52).

IRAK-1 is a key mediator of the IL-1 receptor superfamily, which includes IL-1, IL-18, and the Toll-like receptors. IL-1 signaling plays a critical role in the immune and inflammatory responses and leads to activation of NFKB and AP-1 (48). It may be interesting to investigate whether the upregulation of IRAK-1 contributes to the constitutive activation of these transcription factors in HL $(23,62)$. Prame, which is specifically expressed in all $4 \mathrm{cHL}$ cell lines, is a common tumor antigen that was originally identified as an antigen on melanoma cells recognized by cytotoxic T cells (44). Since Prame is not expressed in normal tissue, it would be a valuable candidate for immunotherapy of cHL.

Taken together, we identified several genes that show strong upregulation in L1236 cells as opposed to their presumed nonmalignant counterpart, GC B cells. Most importantly, for 12 of these genes, it was shown that they are consistently upregulated in cHL cell lines. For 3 genes, expression in primary HRS cells was demonstrated. Of particular interest is the upregulation of several transcription factors and oncogenes that may contribute to HRS cell transformation. It would be interesting to inactivate these factors in HL cell lines or ectopically express them in B cells, in order to assess their functional importance and potential suitability as therapeutic targets. Additionally, some of these factors may become novel prognostic markers.

\section{ACKNOWLEDGMENTS}

This work was supported through the Deutsche Forschungsgemeinschaft by SFB502 and a Heisenberg award to RK. We are grateful to Yvonne Blum, Michaela Fahrig, Christine Gerhardt, and Tanja Schaffer for excellent technical assistance, to Berit Jungnickel for stimulating discussions, and to Carel van Noesel for critically reading this manuscript.

Address correspondence and reprint requests to Ines Schwering, University of Cologne, Department of Internal Medicine I, LFI, E4, R706, Joseph-Stelzmannstr 9, 50931 Cologne, Germany. Phone: +4922 1-478-4490; fax: +49-22 1-478-6383; e-mail: Ines.Schwering@medizin. uni-koeln.de.

Submitted January 3, 2003; accepted for publication February 4, 2003.

\section{REFERENCES}

1. Drexler HG. (1992) Recent results on the biology of Hodgkin and Reed-Sternberg cells. I. biopsy material. Leuk. Lymphoma 8:283-313.

2. Pinkus GS et al. (1997) Fascin, a sensitive new marker for Reed-Sternberg cells of Hodgkin's disease. Evidence for a dendritic or B cell derivation? Am. J. Pathol. 150:543-62.

3. van den Berg A, Visser L, Poppema S. (1999) High expression of the CC chemokine TARC in Reed-Sternberg cells. A possible explanation for the characteristic T-cell infiltration Hodgkin's lymphoma. Am. J. Pathol. 154:1685-91.

4. Küppers R. (2002) Molecular biology of Hodgkin's lymphoma. Adv. Cancer Res. 84:277-312.

5. Stein $\mathrm{H}$ et al. (2001) Down-regulation of BOB.1/OBF.1 and Oct2 in classical Hodgkin disease but not in lymphocyte predominant Hodgkin disease correlates with immunoglobulin transcription. Blood 97:496-501.

6. Refer to http://www.sagenet.org

7. Schwering let al. (2003) Loss of the B lineage-specific gene expression program in Hodgkin and Reed-Sternberg cells of Hodgkin's lymphoma. Blood 101:1505-12.

8. Kanzler H, Küppers R, Hansmann ML, Rajewsky K. (1996) Hodgkin and ReedSternberg cells in Hodgkin's disease represent the outgrowth of a dominant tumor clone derived from (crippled) germinal center B cells. J. Exp. Med. 184:1495-505.

9. Marafioti T et al. (2000) Hodgkin and Reed-Sternberg cells represent an expansion of a single clone originating from a germinal center B-cell with functional immunoglobulin gene rearrangements but defective immunoglobulin transcription. Blood 95:1443-50.

10. Bräuninger A et al. (1999) Identification of common germinal-center B-cell precursors in 2 patients with both Hodgkin's disease and Non-Hodgkin's lymphoma. N. Engl. J. Med. 340:1239-47.

11. Küppers $\mathrm{R}$ et al. (2001) Common germinal-center B-cell origin of the malignan cells in two composite lymphomas, involving classical Hodgkin's disease and either follicular lymphoma or B-CLL. Mol. Med. 7:285-92.

12. Marafioti T et al. (1999) Classical Hodgkin's disease and follicular lymphoma originating from the same germinal center B cell. J. Clin. Oncol. 17:3804-9.

13. Irsch J et al. (2001) Class switch recombination was specifically targeted to immunoglobulin (Ig)G4 or IgA in Hodgkin's disease-derived cell lines. Br. J. Haematol. 113:785-93.

14. Müschen M et al. (2000) Rare occurrence of classical Hodgkin's disease as a T cell lymphoma. J. Exp. Med. 191:387-94.

15. Seitz $V$ et al. (2000) Detection of clonal T-cell receptor gamma-chain gene rearrangements in Reed-Sternberg cells of classic Hodgkin disease. Blood 95:3020-4

16. Cossman J et al. (1999) Reed-Sternberg cell genome expression supports a B-cell lineage. Blood 94:41 1-6.

17. Nacht $M$ et al. (2001) Molecular characteristics of non-small cell lung cancer. Proc. Natl. Acad. Sci. U.S.A. 98:15203-8. 
18. Zhang L et al. (1997) Gene expression profiles in normal and cancer cells. Science 276:1268-72

19. Wolf $J$ et al. (1996) Peripheral blood mononuclear cells of a patient with advanced Hodgkin's lymphoma give rise to permanently growing HodgkinReed Sternberg cells. Blood 87:3418-28.

20. Kanzler $\mathrm{H}$ et al. (1996) Molecular single cell analysis demonstrates the derivation of a peripheral blood-derived cell line (L1236) from the Hodgkin/ReedSternberg cells of a Hodgkin's lymphoma patient. Blood 87:3429-36.

21. Hopken UE et al. (2002) Up-regulation of the chemokine receptor CCR7 in classical but not in lymphocyte-predominant Hodgkin disease correlates with distinct dissemination of neoplastic cells in lymphoid organs. Blood 99:1 109-16.

22. Kapp U et al. (1999) Interleukin 13 is secreted by and stimulates the growth of Hodgkin and Reed-Sternberg cells. J. Exp. Med. 189:1939-46.

23. Bargou RC et al. (1997) Constitutive nuclear factor-kB-RelA activation is required for proliferation and survival of Hodgkin's disease tumor cells. J. Clin. Invest. 100:2961-9.

24. Drexler HG. (1993) Recent results on the biology of Hodgkin and Reed-Sternberg cells. II. continuous cell lines. Leuk. Lymphoma 9:1-25.

25. Velculescu VE, Zhang L, Vogelstein B, Kinzler KW. (1995) Serial analysis of gene expression. Science 270:484-7

26. Klein $U$ et al. (2003) Transcriptional analysis of the B-cell germinal center reaction. Proc. Natl. Acad. Sci. U.S.A. 100:2639-44.

27. Pascual $\vee$ et al. (1994) Analysis of somatic mutation in five $B$ cell subsets of human tonsil. J. Exp. Med. 180:329-39.

28. Oka K, Mori N, Haimoto H, Kato K. (1990) Expression of enolases in T cell tumors and Hodgkin's disease. Lab. Invest. 63:792-7.

29. Messineo C et al. (1998) Gene expression by single Reed-Sternberg cells: pathways of apoptosis and activation. Blood 91:2443-51.

30. Anwar F, Wood BL. (2000) CD44H and CD44V6 expression in different subtypes of Hodgkin lymphoma. Mod. Pathol. 13:1121-7.

31. Johnson EN, Druey KM. (2002) Functional characterization of the G protein regulator RGS13. J. Biol. Chem. 277:16768-74.

32. Zhou J et al. (2001) Characterization of RGS5 in regulation of $G$ protein-coupled receptor signaling. Life Sci. 68:1457-69.

33. Spain BH et al. (1996) Two human CDNAs, including a homolog of Arabidopsis FUS6 (COP1 1), suppress G-protein- and mitogen-activated protein kinase-mediated signal transduction in yeast and mammalian cells. Mol. Cell Biol. 16:6698706.

34. Bachner D, Sedlacek Z, Korn B, Hameister H, Poustka A. (1995) Expression patterns of two human genes coding for different rab GDP-dissociation inhibitors (GDIs), extremely conserved proteins involved in cellular transport. Hum. Mol. Genet. 4:701-8.

35. Scherle P, Behrens T, Staudt LM. (1993) Ly-GDI, a GDP-dissociation inhibitor of the RhoA GTP-binding protein, is expressed preferentially in lymphocytes. Proc. Natl. Acad. Sci. U.S.A. 90:7568-72.

36. Saha $S$ et al. (2001) A phosphatase associated with metastasis of colorectal cancer. Science 294:1343-6.

37. Clark EA, Golub TR, Lander ES, Hynes RO. (2000) Genomic analysis of metastasis reveals an essential role for rhoC. Nature 406:532-5.

38. Barrett J, Birrer MJ, Kato GJ, Dosaka-Akita H, Dang CV. (1992) Activation domains of L-Myc and C-Myc determine their transforming potencies in rat embryo cells. Mol. Cell Biol. 12:3130-7.

39. Lah TT, Kos J. (1998) Cysteine proteinases in cancer progression and their clinical relevance for prognosis. Biol. Chem. 379:125-30.

40. Menard S, Tagliabue E, Colnaghi MI. (1998) The $67 \mathrm{kDa}$ laminin receptor as a prognostic factor in human cancer. Breast Cancer Res. Treat. 52:137-45.

41. Goodison S, Urquidi V, Tarin D. (1999) CD44 cell adhesion molecules. Mol. Pathol. 52:189-196.

42. Boutros M, Mlodzik M. (1999) Dishevelled: at the crossroads of divergent intracellular signaling pathways. Mech. Dev. 83:27-37.

43. Aryee DN et al. (1996) Variability of nm23-HI/NDPK-A expression in human lymphomas and its relation to tumour aggressiveness. Br. J. Cancer 74:1693-8.

44. Ikeda $\mathrm{H}$ et al. (1997) Characterization of an antigen that is recognized on a melanoma showing partial HLA loss by CTL expressing an NK inhibitory receptor. Immunity 6:199-208.

45. Iacobelli M, Wachsman W, McGuire KL. (2000) Repression of IL-2 promoter activity by the novel basic leucine zipper p21SNFT protein. J. Immunol. 165:860-8.

46. Peters CS et al. (2001) ATF-7, a novel bZIP protein, interacts with the PRL-1 protein-tyrosine phosphatase. J. Biol. Chem. 276:13718-26.

47. Morinaga T, Yasuda H, Hashimoto T, Higashio K, Tamaoki T. (1991) A human alpha-fetoprotein enhancer-binding protein, ATBF1, contains 4 homeodomains and seventeen zinc fingers. Mol. Cell Biol. 11:6041-9.

48. Li X, Commane M, Jiang Z, Stark GR. (2001) IL-1-induced NFא B and C-Jun N-terminal kinase (JNK) activation diverge at IL-1 receptor-associated kinase (IRAK) Proc. Natl. Acad. Sci. U.S.A. 98:4461-5.

49. Bolin LM et al. (1997) HNMP-1: a novel hematopoietic and neural membrane protein differentially regulated in neural development and injury. J. Neurosci. 17:5493-502.

50. Guzman-Rojas L et al. (2000) PRELI, the human homologue of the avian px19 is expressed by germinal center B lymphocytes. Int. Immunol. 12:607-12.

51. Peattie DA et al. (1992) Expression and characterization of human FKBP52, an immunophilin that associates with the 90-kDa heat shock protein and is a component of steroid receptor complexes. Proc. Natl. Acad. Sci. U.S.A 89:10974-8.

52. Shaulian E, Karin M. (2001) AP-1 in cell proliferation and survival. Oncogene 20:2390-2400

53. Küppers $R$ et al. (2003) Identification of Hodgkin and Reed-Sternberg cellspecific genes by gene expression profiling. J.Clin. Invest. 111:529-37.

54. Jox A et al. (1999) Somatic mutations within the untranslated regions of rearranged Ig genes in a case of classical Hodgkin's disease as a potential cause for the absence of Ig in the lymphoma cells. Blood 93:3964-72.

55. Buckbinder $\mathrm{L}$ et al. (1997) The $\mathrm{p} 53$ tumor suppressor targets a novel regulator of G protein signaling. Proc. Natl. Acad. Sci. U.S.A. 94:7868-72.

56. Aron B, Hall A. (2002) Rho GTPases in Transformation and Metastasis. Adv. Cancer Res. 84:57-80.

57. Cates CA et al. (1996) Prenylation of oncogenic human PTP(CAAX) protein tyrosine phosphatases. Cancer Lett. 110:49-55.

58. Diamond RH, Cressman DE, Laz TM, Abrams CS, Taub R. (1994) PRL-1, a unique nuclear protein tyrosine phosphatase, affects cell growth. Mol. Cell Biol. 14:3752-62.

59. Kaspar $P$ et al. (1999) Myb-interacting protein, ATBF1, represses transcriptiona activity of Myb oncoprotein. J. Biol. Chem. 274:14422-8.

60. Berry FB et al. (2001) Positive and negative regulation of myogenic differentiation of $\mathrm{C} 2 \mathrm{C} 12$ cells by isoforms of the multiple homeodomain zinc finger transcription factor ATBF1. J. Biol. Chem. 276:25057-65.

61. Miura $Y$ et al. (1995) Cloning and characterization of an ATBFl isoform that expresses in a neuronal differentiation-dependent manner. J. Biol. Chem. 270:26840-8.

62. Mathas S et al. (2002) Aberrantly expressed C-Jun and JunB are a hallmark of Hodgkin lymphoma cells, stimulate proliferation and synergize with NF-кB. EMBO J. 21:4104-13. 